\title{
BMJ Open Economic evaluation alongside the Probiotics to Prevent Severe Pneumonia and Endotracheal Colonization Trial (E- PROSPECT): study protocol
}

\author{
Vincent Issac Lau (D) ,,2 Deborah J Cook, ${ }^{2,3}$ Robert Fowler, ${ }^{4}$ Bram Rochwerg (D) ,2,3 \\ Jennie Johnstone, ${ }^{5}$ François Lauzier, ${ }^{6}$ John C Marshall, ${ }^{7}$ John Basmaji, ${ }^{8}$ \\ Diane Heels-Ansdell, ${ }^{2,9}$ Lehana Thabane, ${ }^{2,9}$ Feng Xie, ${ }^{2,9}$ PROSPECT Collaborators
}

To cite: Lau VI, Cook DJ, Fowler R, et al. Economic evaluation alongside the Probiotics to Prevent Severe Pneumonia and Endotracheal Colonization Trial (E-PROSPECT): study protocol. BMJ Open 2020;10:e036047. doi:10.1136/ bmjopen-2019-036047

- Prepublication history and additional material for this paper are available online. To view these files, please visit the journal online (http://dx.doi. org/10.1136/bmjopen-2019036047).

Received 29 November 2019 Revised 19 May 2020 Accepted 20 May 2020

Check for updates

(C) Author(s) (or their employer(s)) 2020. Re-use permitted under CC BY-NC. No commercial re-use. See rights and permissions. Published by BMJ.

For numbered affiliations see end of article.

Correspondence to

Dr Vincent Issac Lau; vinceissaclau@gmail.com

\section{ABSTRACT}

Introduction Ventilator-associated pneumonia (VAP) is a common healthcare-associated infection in the intensive care unit (ICU). Probiotics are defined as live microorganisms that may confer health benefits when ingested. Prior randomised trials suggest that probiotics may prevent infections such as VAP and Clostridioides difficile-associated diarrhoea (CDAD). PROSPECT (Probiotics to Prevent Severe Pneumonia and Endotracheal Colonization Trial) is a multicentre, double-blinded, randomised controlled trial comparing the efficacy of the probiotic Lactobacillus rhamnosus GG with usual care versus usual care without probiotics in preventing VAP and other clinically important outcomes in critically ill patients admitted to the ICU.

Methods and analysis The objective of E-PROSPECT is to determine the incremental cost-effectiveness of $L$. rhamnosus GG plus usual care versus usual care without probiotics in critically ill patients. E-PROSPECT will be performed from the public healthcare payer's perspective over a time horizon from ICU admission to hospital discharge.

We will determine probabilities of in-ICU and in-hospital events from all patients alongside PROSPECT. We will retrieve unit costs for each resource use item using jurisdiction-specific public databases, supplemented by individual site unit costs if such databases are unavailable. Direct costs will include medications, personnel costs, radiology/laboratory testing, operative/non-operative procedures and per-day hospital 'hoteling' costs not otherwise encompassed. The primary outcome is the incremental cost per VAP prevented between the two treatment groups. Other clinical events such as CDAD, antibiotic-associated diarrhoea and in-hospital mortality will be included as secondary outcomes. We will perform pre-specified subgroup analyses (medical/surgical/trauma; age; frailty status; antibiotic use; prevalent vs no prevalent pneumonia) and probabilistic sensitivity analyses for VAP, then generate confidence intervals using the nonparametric bootstrapping approach.

Ethics and dissemination Study approval for EPROSPECT was granted by the Hamilton Integrated Research Ethics Board of McMaster University on 29 July 2019. Informed consent was obtained from the patient or substitute decision-maker in PROSPECT. The
Strengths and limitations of this study

- A priori study protocol with prospective clinical and economic data collection with representation from international jurisdictions.

- The balance of randomisation reduces risk of bias in the cost-effectiveness analysis occurring on patient level.

- A relatively short time horizon.

- Primary outcome of incremental cost to avoid a clinical event (cost-effectiveness approach), rather than a cost-utility approach (incremental cost per qualityadjusted life year).

findings of this study will be published in peer-reviewed journals.

Trial registration number NCT01782755; Pre-results.

\section{BACKGROUND}

Context

Ventilator-associated pneumonia (VAP) is the most common healthcare-associated infection in the intensive care unit (ICU), resulting in a high burden of illness. ${ }^{12}$ A 2005 systematic review found a pooled cumulative VAP incidence of $23 \%$ (95\% CI $19 \%$ to $27 \%$ ) in randomised controlled trials (RCTs) and $10 \%(95 \%$ CI $7 \%$ to $13 \%)$ in observational studies. $^{2}$ In addition, VAP is associated with a twofold attributable risk of dying in the ICU (OR 2.02, 95\% CI 1.2 to 3.6), and the cost attributed to VAP ranges from US\$10 000 to $\$ 13000$ per patient. ${ }^{2}$ Thus, VAP prevention is a patient-important safety goal during critical illness. 134

Probiotics are defined as "live microorganisms which, when administered in adequate amounts, may confer a potential health benefit on the host". ${ }^{5} 6$ They are reported to enhance gut barrier function, reduce host pathogenic bacterial load, modify gut 
microbiota and modulate the immune system. ${ }^{7-10}$ Probiotics studies suggest benefits including reduced incidence of healthcare-associated infections. ${ }^{1-14} \mathrm{~A}$ recent meta-analysis of RCTs suggests that probiotics administered to critically ill mechanically ventilated patients were associated with a $26 \%$ lower VAP rate $(95 \%$ CI $10 \%$ to $39 \%$ ) and $20 \%$ lower infection rates overall $(95 \%$ CI $5 \%$ to $32 \%) \cdot{ }^{15}$ However, these findings arose from 30 small, mostly low-quality single-centre RCTs ( $\mathrm{n}=18-300,2972$ total patients in the meta-analysis), yielding imprecise estimates and results with uncertain internal and external validity. ${ }^{15}$

Further, probiotics may reduce the incidence of diarrhoea, specifically Clostridioides difficile-associated diarrhoea (CDAD), which can cause serious complications such as pseudomembranous colitis, toxic megacolon and death. ${ }^{16}$ In a recent Cochrane systematic review and metaanalysis of 31 RCTs including 8672 patients who were receiving antibiotics and concurrent probiotics, moderate certainty evidence suggested that probiotics were effective at reducing the burden of CDAD for patients and the healthcare system. ${ }^{16}$

\section{Current Knowledge}

We recently performed a systematic review of economic evaluations examining probiotics in hospitalised patients, evaluating their cost-effectiveness for reducing VAP, CDAD and antibiotic-associated diarrhoea (AAD), while also identifying variables that could drive costs. ${ }^{17}$ From 721 potentially relevant studies, 7 met the eligibility criteria. Probiotics appear to be either cost-effective or cost-saving in six of seven studies compared with other prophylactic strategies within usual care to prevent healthcare-associated infection in acutely ill hospitalised patients. However, Grading of Recommendations Assessment, Development and Evaluation (GRADE) evaluations indicated a high risk of bias and very low quality/ certainty of clinical evidence, such that cost-effectiveness evidence on the use of probiotics in adult hospitalised patients was weak. Furthermore, probiotic manufacturers funded three of seven (43\%) studies, all of which were reported as either cost-effective or cost-saving. ${ }^{17}$ Some probiotic economic evaluations were designed after the results of the trial were published.

\section{Study Aims}

Therefore, we have designed this economic evaluation (E-PROSPECT) alongside the multicentre PROSPECT ( ClinicalTrials.gov no. NCT01782755), assessing the incremental cost-effectiveness ratio (ICER) of probiotics versus usual care for critically ill adult patients. ${ }^{18-20}$

\section{METHODS}

\section{Overview of prospect}

PROSPECT is a randomised, double-blinded multicentre controlled trial. It used a central system for concealed 1:1 ratio to randomise patients (in variable unspecified block sizes, stratified by centre and by medical, surgical or trauma admission status) to either $1 \times 10^{10}$ colony-forming units of Lactobacillus rhamnosus GG (iHealth, Inc.) or an identical placebo suspended in tap water administered twice daily via feeding tube in the ICU. ${ }^{20}$ PROSPECT has enrolled 2653 critically ill patients between October 2013 and March 2019 throughout 44 ICUs (41 in Canada, 2 in the USA and 1 in Saudi Arabia). Patients, healthcare providers, investigators and research personnel were all blinded to group allocation. Sample size calculation has been previously described. ${ }^{18-20}$

\section{E-PROSPECT design}

The primary objective of E-PROSPECT is to estimate the incremental cost per VAP prevented arising from a prevention strategy of using probiotics with usual care (the probiotics arm) versus usual care without probiotics (the usual care arm) during hospitalisation. Our secondary analyses of ICERs include healthcare-associated complications (CDAD, AAD) and mortality. ${ }^{18-20}$

Our economic evaluation will be performed from the public healthcare payer's perspective, ${ }^{21}$ over the time horizon of the ICU admission to hospital discharge or death (table 1). Our economic evaluation protocol was developed (table 1) according to established CHEERS (Consolidated Health Economic Evaluation Reporting Standards) and international cost-effectiveness analysis guidelines. $^{22} 23$

\section{Clinical outcomes}

Clinical outcomes that will be examined in E-PROSPECT are described with definitions in online supplementary table 1 that were previously described from PROSPECT. ${ }^{20}$ Clinical events such as VAP (primary outcome), CDAD, $\mathrm{AAD}$ and hospital mortality (secondary outcomes) will be gleaned from PROSPECT, with a statistical analysis methodology previously described. ${ }^{20}$ For the dichotomous outcomes, we will use time-to-event analyses. HRs and associated $95 \%$ CIs will be estimated using a stratified Cox proportional-hazards model. For continuous outcomes, we will report estimates of the difference between intervention and control groups, 95\% CIs and associated $\mathrm{p}$ values. $^{20}$

These dichotomous outcomes with proportions and continuous outcomes with point estimates (eg, length of stay, which will be used for calculation of resource utilisation) will be used to calculate both incremental costs (resource utilisation) and effects. Incremental effects will be defined as the difference in per-patient event rates or the difference in proportion of a clinical event (eg, VAP) between groups.

\section{Health care resource utilisation}

Based on our systematic literature review ${ }^{17}$ and published evidence, ${ }^{18-20}$ we identified a list of relevant healthcare resource items that includes medications, physician/ personnel utilisation, diagnostic radiology/laboratory testing, and operative/non-operative procedures and 
Table 1 Summary of economic evaluation framework

Question Is the use of probiotics as compared with standard care without probiotics cost-effective for the prevention of VAP and other clinically important outcomes in critically ill medical-surgical patients in PROSPECT?

\begin{tabular}{ll} 
Perspective & Public payer (in-hospital costs) \\
\hline Setting & Ventilated ICU patients (44 centres, 3 countries: 41 Canada, 2 USA, 1 Saudi Arabia) \\
\hline Comparators & Probiotics (Lactobacillus rhamnosus GG) with usual care vs usual care without probiotics \\
\hline Time horizon & From ICU participant admission to hospital discharge/death (non-fixed time span) \\
Discount rate & No discounting (no long-term follow-up over 1 year) \\
Clinical outcomes & VAP, CDAD, AAD, length of stay and mortality (ICU and hospital) \\
Costs & Direct medical costs associated with treatment and complications (ICU and ward costs, personnel, \\
medications, laboratory tests, diagnostic testing and procedures/surgeries)
\end{tabular}

AAD, antibiotic associated diarrhoea; CDAD, Clostriodiodes difficile-associated diarrhoea; ICER, incremental cost-efficacy/effectiveness ratio; ICU, intensive care unit; PROSPECT, Probiotics: Prevention of Severe Pneumonia and Endotracheal Colonization Trial; VAP, ventilatorassociated pneumonia.

per-day hospital 'hoteling' costs not otherwise encompassed. Antimicrobial use in ICU will be defined as days of therapy, defined daily dose of therapy and antimicrobialfree days. $^{24} 25$ Only systemic antimicrobials will be captured whether prophylactic or therapeutic in intent. Topical creams, eye/ear drops and inhaled antimicrobials will be excluded. We will also document the duration of mechanical ventilation, ICU and hospital length of stay and mortality. The healthcare resource uses will be collected alongside PROSPECT. For missing resource use data, we will choose appropriate imputation methods according to the type and distribution of the missing data. $^{2627}$ Otherwise, we will use an appropriate 'standard dose' for non-titratable medications (eg, chlorhexidine) and a clinically appropriate 'medium dose' for titratable medications (eg, vasopressors or inotropes).

\section{Unit costs}

Unit costs for healthcare resource items will be identified through jurisdiction-specific (regions/provinces/states which manage healthcare delivery in their area) public databases (eg, pharmacy drug formularies, physician billing schedule of benefits, Medicare/Medicaid reimbursement manuals, labour department wages/salaries, manufacturer costs). When there is a small sample or distribution of unit costs (ie, a provincial jurisdiction may have the same cost for a particular procedure), we will estimate the SE if possible, or incorporate a $\pm 25 \%$ error around the mean unit cost distribution.
For unit costs not represented in public databases, we will obtain site-specific unit costs from the participating PROSPECT sites. We will first conduct a pilot study of unit cost acquisition at a convenience sample of nine participating centres (Canadian: British Columbia, Alberta, Manitoba, Ontario, Quebec, Nova Scotia; US: Minnesota, Missouri; and Saudi Arabia) to request a list of unit costs (online supplementary table 2: E-PROSPECT unit cost data extraction table). The site investigator or research co-ordinator will then contact the most appropriate individual in each hospital's accounting, human resources, pharmacy, radiology or laboratory departments to obtain the unit costs. ${ }^{28}$ In all cases, costs will be requested (if available). If only charges are known, then we will attempt to convert to costs by the institution's cost-to-charge estimate for that item, where it exists. ${ }^{28}$

Direct costs will be presented in the pre-specified cost categories (online supplementary table 2). Assumptions regarding resource utilisation are presented in online supplementary table 3 . We will assess direct unit costs for study product-related resources associated with outcomes of VAP, CDAD, AAD and mortality. If a specific line-item unit cost is not attainable for a specific jurisdiction, ${ }^{28}$ we will (1) ask another site within the same jurisdiction for missing unit costs and (2) derive a cost-ratio from acquired line-items (ie, drug costs both known in two jurisdictions), then using the cost-ratio impute the missing line-item unit costs for the missing jurisdiction (by multiplying the cost-ratio against a known 
jurisdiction's acquired line-item to impute the line-item unit cost for the missing jurisdiction). (3) If line-item unit costs are still missing after multiple imputation (with missing variables), a mean unit cost approach will be used for the remaining jurisdictions which did report unit costs.

The pilot phase may inform amendments to our protocol. For example, if a unit cost for a particular line-item is deemed to be small and/or has a low clinical incidence rate, then that line-item may be removed from the final analysis. Items without a difference in clinical outcome/resource utilisation between intervention and control groups but which contribute substantially to costs may still be retained (even if little to no incremental difference in costs would exist between the two arms) in order to maintain face validity and accurately reflect the magnitude of costs for hospitalisation of a critically ill patient. Once the list of line-items has been pared down to those which are deemed to be cost drivers, and clinically relevant while also feasible to obtain, the remaining line-item list will be surveyed across a sampling of individual sites from each representative jurisdiction from PROSPECT.

Unit cost data will be summarised among all sites, and by country, to explore variability across centres and countries and to improve the generalisability of results. Visible outliers will be reconfirmed with individual hospital contacts. Participating sites will be queried to determine if particular costs have changed substantially (eg, by more than $25 \%$ ), beyond inflationary or deflationary changes, over the course of the study. If there are substantial changes that have occurred over time, we will use the mean unit costs adjusted for inflation over the mean duration of the trial. ${ }^{28}$

\section{Cost analysis}

The cost for each resource use item will be calculated by multiplying the natural resource utilisation units by the unit cost. The total cost per patient will be the sum of the cost of items used from the time of randomisation until discharge from hospital or death. The incremental mean cost will be estimated by calculating the difference in the total per-patient costs between the two groups. All costs will be converted to 2019 US dollars, accounting for annual inflation. ${ }^{29-33}$

We plan on using international currency conversion, instead of purchase power parity (PPP)-based conversions, because health-specific PPPs are not available for all participating countries, and non-health PPP conversion rates vary substantially over the period of the analysis. ${ }^{30}$ Country-specific costs will be considered only in sensitivity analyses.

Incremental costs will be calculated using the difference in mean per-patient cost between the two treatment arms. We have developed a costing operations manual outlining this process (online supplementary table 4: E-PROSPECT costing manual). ${ }^{30}$

\section{Cost-effectiveness analyses}

Means (SD) or frequency (percentage) will be used to describe effect and cost estimates wherever appropriate. Chi-square tests and two-sample t-test comparisons will be used as appropriate to compare baseline characteristics between the two arms. The primary outcome will be based on the intention-to-treat principle and will form the clinical event estimates for the economic evaluation. Regression analyses may be performed if there is residual confounding, based on previously described methodology. ${ }^{20}$

The incremental cost-effectiveness ratio (ICER) is the ratio of incremental costs per VAP prevented of probiotics versus usual care during the period of hospitalisation (from ICU admission to hospital discharge or death). The incremental mean costs will be estimated from all patients in both groups based on multiplying the resource unit cost by resource utilisation as described previously. The incremental mean effects will be derived from PROSPECT, where incremental effects were defined as the difference in per-patient event rates or the difference in proportion of a clinical event (eg, VAP) between groups. ${ }^{28}{ }^{34}$ In secondary analysis, we will also calculate ICER using other clinical outcomes (ie, CDAD, AAD, mortality). If there is dominance in cost-effectiveness (ie, one treatment is better at lower cost than the other treatment), we will present the difference in cost and effect separately, without calculating the ICER for the base-case analysis. When there is no difference in clinical outcomes, we will present incremental cost and effects separately, without calculating an ICER for the base-case analysis.

\section{Subgroup analyses}

As subgroup analyses, we will investigate specific patients who may have differential effects and costs as compared with the entire population, including diagnostic category (medical, surgical, trauma) ${ }^{2}$; age $<65$ years, $65-75$ years and $>75$ years $^{35}{ }^{36}$; frailty status (baseline Clinical Frailty Score $\geq 5$ of 9 vs 5$)^{37}$; patients who received/did not receive antibiotics within 2 days of randomisation ${ }^{20}$; and prevalent (present at the time of enrolment) versus no prevalent pneumonia. ${ }^{20}$

\section{Uncertainty analyses}

Because patient characteristics and costs may differ in different jurisdictions and outside clinical trial settings, and there will be uncertainty associated in the estimation of each group's clinical outcomes and separately in the associated group's costs, we have prospectively planned an uncertainty analysis to explore how ICERs may change with plausible ranges in costs of probiotics.

To test the robustness of our results (and determine the uncertainty associated with cost and effects estimation), we will perform a probabilistic sensitivity analysis of pairs of known costs and effects, using non-parametric bootstrapping techniques to generate $95 \%$ CIs. We will perform 1000 bootstrap simulations in the following manner: each simulation will draw the same number of patients 
per group (as per intention-to-treat), with replacement (for both events and cost) in pairs. For each sample, the difference in event rate and cost was calculated, obtaining 1000 pairs of differences in cost and event rate. ${ }^{38}{ }^{39}$ Costeffectiveness acceptability curves will be used to present the probability of probiotics being cost-effective over a wide range of willingness-to-pay thresholds. ${ }^{21}$

Scenario analyses will also be performed with variations of estimates of pairs of potentially influential variables (ie, costs of probiotics, per-day cost of care in ICU and hospital wards) across plausible ranges (variation of costs: $50 \%-150 \%$ ) to explore potential cost differences in higher-spending and lower-spending healthcare jurisdictions to determine if different estimates change the overall results.

All analyses will be undertaken using Excel (Microsoft, Redmond, Washington, USA) and SAS (Cary, North Carolina, USA).

\section{Patient and public involvement}

Patients or the public were not involved in the development of the research question, design, or conduct, or reporting, or dissemination plans of our research. The burden of the intervention was not assessed in the patients themselves.

\section{Ethics and dissemination}

Research ethics approval for E-PROSPECT was granted by the Hamilton Integrated Research Ethics Board (HIREB) of McMaster University (project identifier: REB\#:15-322). Informed consent was obtained from each participant in PROSPECT, or their substitute decisionmaker, in accordance with local REB approvals. We anticipate that a majority of sites participating in E-PROSPECT will consider central HIREB approval as satisfactory to obtain additional non-specific patient-based costing data from their centre. All economic data, as with trial data, will be de-identified, maintained in a password-protected and encrypted laptop or desktop, in locked offices. All de-identified datasets, technical appendices and statistical code will be published alongside the economic evaluation. Knowledge translation of the results will be disseminated to patients, public and healthcare providers through peer-reviewed journals. The CHEERS checklist has been completed (online supplementary table 5).

\section{DISCUSSION}

PROSPECT is the largest trial undertaken of probiotic usage for VAP prophylaxis in critically ill patients. Although probiotics have been shown in prior trials to prevent VAP and CDAD, their relative effects, side effects and cost-effectiveness remain uncertain. PROSPECT will determine whether probiotics reduce the frequency of VAP and other healthcare-associated complications during critical illness. ${ }^{18-20}$

An economic evaluation jointly considers both costs and effects between alternative treatment options. Thus, physicians, administrators and policy-makers can know whether a new treatment provides good value for the healthcare expenditure. E-PROSPECT will answer these questions and address the cost-effectiveness of probiotics for VAP prevention. The literature currently has a paucity of health economic evaluations, illustrating the importance of E-PROSPECT. ${ }^{40}$

\section{Strengths and limitations}

Some aspects of our methodology have potential limitations. First, the time horizon is relatively short, with no outpatient follow-up (only reporting in-hospital outcomes). Other studies have used relative, nonfixed time horizons in health economic evaluations, ${ }^{34}$ including those investigating probiotics. ${ }^{41} 42$ We will carefully interpret these cost-effectiveness ratios in context from the short time horizon. Second, our primary outcome is the incremental cost to avoid a VAP event and other clinically important outcomes, not the incremental cost per quality-adjusted life year gained in a cost-utility analysis. ${ }^{21}$ PROSPECT is not designed to measure longterm outcome or downstream life expectancy (hence no lifetime time horizon). However, if PROSPECT shows a difference in hospital survival due to probiotics, this will be addressed as a secondary outcome. As with all efficacy trials, the generalisability and external validity of a health economic evaluation concurrently performed with an RCT may not represent the same treatment effects and costs as in routine clinical practice.

E-PROSPECT has several advantages. ${ }^{43}$ First, we reduce the potential for investigator hypothesis-driven biases by pre-specifying our parameters of analysis (subgroup and sensitivity analysis) for the health economic evaluation prior to unblinding of the trial. Second, trial randomisation can reduce bias and confounding according to different baseline characteristics between study groups. Third, the concurrent collection of clinical and economic data can reduce the costs of data collection and minimise the possible problem of missing data if attempting to obtain it retrospectively. Fourth, we have chosen to gather costs from healthcare systems from multiple countries participating in the PROSPECT trial. We anticipate a wide variability in institutional reporting patient-specific cost accounting. ${ }^{28} 34$ Although this has the potential to introduce variability in cost estimates, this approach will also likely enhance the generalisability of our results. Finally, timely economic data can be useful to healthcare policy-makers to aid in resource allocation decisions. There are several clinician-researchers that are advocating for the embracing the science of value in healthcare, ${ }^{44}$ while others state that cost-effectiveness analysis should be mandatory in clinical-effectiveness research to aid in clinical guideline development and public healthcare decision policy. ${ }^{45}$ By conducting our economic analysis concurrent with the PROSPECT trial, we take advantage of each of these strengths. ${ }^{28}$

\section{Author affiliations}

${ }^{1}$ Department of Critical Care, University of Alberta Faculty of Medicine and Dentistry, Edmonton, Alberta, Canada 
${ }^{2}$ Department of Health Research Methods, Evidence and Impact, McMaster University, Hamilton, Ontario, Canada

${ }^{3}$ Division of Critical Care, McMaster University, Hamilton, Ontario, Canada ${ }^{4}$ Sunnybrook Health Sciences Institute, Sunnybrook Research Institute, Toronto, Ontario, Canada

${ }^{5}$ Public Health Ontario, University of Toronto Dalla Lana School of Public Health, Toronto, Ontario, Canada

${ }^{6}$ Population Health and Optimal Health Practices Research Unit (TraumaEmergency-Critical Care Medicine), Centre de Recherche du CHU de QuébecUniversité Laval, Quebec, Quebec, Canada

${ }^{7}$ Department of Surgery, University of Toronto, Toronto, Ontario, Canada ${ }^{8}$ Department of Medicine, Division of Critical Care, Western University, London, Ontario, Canada

${ }^{9}$ Clinical Epidemiology and Biostatistics, McMaster University, Hamilton, Ontario, Canada

\section{Twitter Bram Rochwerg @bramb043}

Acknowledgements PROSPECT was designed by the PROSPECT Steering Committee and improved by Drs Dawn Bowdish, Michael Surette and Erick Duan, the PROSPECT Investigators and Research $\mathrm{Co}$-ordinators and the Canadian Critical Care Trials Group. We are grateful for the commitment of all our colleagues in participating centres, and staff at the Methods Center for their expertise including Nicole Zytaruk, Lois Saunders, Shelley Anderson-White, Mary Copland, Megan Davis, France Clarke and Alyson Takaoka.

Collaborators PROSPECT Collaborators: Canada-St Joseph's Healthcare, Hamilton, ON: Dr Deborah Cook (Lead); Dr Erick Duan, Dr Mark Soth (CoInvestigators); France Clarke, Mary Copland; Neala Hoad, Marnie Jakab, Melissa Shears, Alyson Takaoka, Nicole Zytaruk (Research Co-ordinators); Christa Connolly, Denise Davis, Catherine Eaton, Tracy Gallinas, Jean Lee-Yoo, Connie Lukinuk, Leia Musielak, Nancy Pavunkovic, Joy Pelayo, Kaitlyn Phillips, Catherine Pracsovics, Julia Raimondo, Vida Stankus, Christine Wallace, Angela Wright, Crystal Young (Pharmacy), Hamilton General Hospital, Hamilton Health Science Center, Hamilton, ON: Dr Maureen Meade (Lead); Dr Emilie Belley-Cote (Co-Investigator), Katrina Fimiani, Lori Hand, Dr Harjot Jagdey, Lisa Klotz, Alexandra Sabev, Nevena Savija (Research Co-ordinators); Deanne Cosentino, Diane Lourenco, Julie Misina, Gita Sobhi (Pharmacy), Juravinski Hospital Hamilton Health Science Centre, Hamilton, ON: Dr Timothy Karachi (Lead), Dr Bram Rochwerg (Co-Investigator); Mashari Alghuroba, Alia Khaled, Lauren Locco, Tina Millen, Ryan Vaisler (Research Co-ordinators); Maya Biljan, Deanne Cosentino, Brittany Marriott, Gita Sobhi (Pharmacy), St Michael's Hospital, Toronto, ON: Dr John Marshall (Lead); Dr Jan Friedrich (Co-Investigator); Jennifer Hodder, Imrana Khalid, Julie Lee, Yoon Lee, Pragma Roy, Kurtis Salway, Gyan Sandhu, Marlene Santos, Orla Smith, Melissa Wang (Research Co-ordinators); Norman Dewhurst, Ann Dowbenka, Ann Kosinski, Terri Norrie, Ranjit Parhar, Laura Parsons, Johanna Proceviat, Gitana Ramonas, Mae Yuen (Pharmacy), St Paul's Hospital, Vancouver BC: Dr Peter Dodek (Lead); Dr Najib Ayas (Co-Investigator); Maria Agda, Victoria Alcuaz, Betty Jean Ashley, Kelsey Brewer, Janice Palmer (Research Co-ordinators); Glen Brown, Mara Pavan (Pharmacy), Mount Sinai Hospital, Toronto, ON: Dr Geeta Mehta (Lead), Dr Stephen Lapinsky, Dr Laveena Munshi; Maedean Brown, Brittany Giacomino, Marnie Jakab, Alan Kraguljac, Sumesh Shah, Erik Tamberg, Laura Vergeer (Research Coordinators); Doret Cheng, Gagan Grewal, Andrew Han, Holly Leung, loanna Mantas, Hilary Rodrigues, Andrew Wyllie (Pharmacy), CHU de Québec-Université Laval (Hôpital de l'Enfant-Jésus), Québec City, QC: Dr François Lauzier (Lead); Dr Alexis Turgeon (Co-Investigator); Danny Barriault, David Bellemare, Anick Boivin, Sarah-Judith Breton, Ėve Cloutier, Marjorie Daigle, Charles Delisle-Thibeault, Panagiota Giannakouros, Stéphanie Grenier, Gabrielle Guilbault, Caroline Léger, Catherine Ouellet, Marie-Claude Tremblay (Research Co-ordinators); Élisabeth Gagne, Julie Gaudreau, Claire Grégoire, Véronique Labbé, Ariane Laprise-Rochette, Caroline Ouellet, Mélanie Samson, Marie-David Simoneau, Virginie Turcotte, Tuong-Vi Tran (Pharmacy), Ottawa Civic Hospital, Ottawa, ON: Dr Lauralyn McIntyre (Lead), Dr Joe Pagilarello (Co-Investigator); Pierre Cardinal, Gianni D'Egidio, Shane English, Mike Hartwick, Jonathon Hooper, Gwynne Jones, John Kim, Dal Kubelik, Kwadwo Kyeremanteng, Hilary Meggison, Sherissa Microys, Dave Neiliovitz, Guiseppe Pagliarello, Rakesh Patel, Jo Po, Peter Reardon, Erin Rosenberg, Aimee Sarti, Andrew Seely (Co-Investigators); Shelley Acres, Brigette Gomes, Heather Langlois, Liane Leclair, Sydney Miezitis, Kaitlyn Montroy, Rebecca Porteous, Shawna Reddie, Irene Watpool (Research Co-ordinators); Wendy Aikens, Marianne Cox, Ann-Marie Dugal, Susan Fetzer, Kathy Fraser, Jennifer Kuhn, Rob MacLeod, Susanne Richard, Dawn Rose, Sherry Weir (Pharmacy), Ottawa General Hospital, Ottawa, ON: Dr Lauralyn McIntyre (Lead), Dr Pierre Cardinal, Dr Gianni D’Egidio, Dr
Shane English, Dr Mike Hartwick, Dr Jonathon Hooper, Dr Gwynne Jones, Dr John Kim, Dr Dal Kubelik, Dr Kwadwo Kyeremanteng, Dr Hilary Meggison, Dr Sherissa Microys, Dr Dave Neiliovitz, Dr Guiseppe Pagliarello, Dr Rakesh Patel, Dr Jo Po, Dr Peter Reardon, Dr Erin Rosenberg, Dr Aimee Sarti, Dr Andrew Seely (CoInvestigators); Shelley Acres, Heather Langlois, Liane Leclair, Sydney Miezitis, Kaitlyn Montroy, Rebecca Porteous, Shawna Reddie, Amanda Van Beinum, Allyshia Van Tol, Irene Watpool (Research Co-ordinators); Wendy Aikens, Anne-Marie Dugal, Susan Fetzer, Sherry Weir (Pharmacy), Vancouver General Hospital, Vancouver, BC: Dr Bill Henderson (Lead), Dr Donald Griesdale, Dr Mypinder Sekhon (CoInvestigators); Denise Foster, Suzie Logie (Research Co-ordinators); Judy Yip (Pharmacy), University Health Network, Toronto Western Hospital, Toronto, ON: Dr Margaret Herridge (Lead), Dr Alberto Goffi, Dr Eyal Golan, Dr John Granton, Dr Victoria McCredie, Dr Elizabeth Wilcox (Co-Investigators); Jaimie Archer, Daniel Chen, Paulina Farias, Brooke Fraser, Cheryl Geen-Smith, Barbara Kosky, Andrea Matte, Christina Pugliese, Priscila Robles, Lia Stenyk, Cristian Urrea, Karolina Walczak (Research Co-ordinators); Kyung Ae, Jane Ascroft, Fatima Haji, Rajvinder Kaur, Jane Lui, Sophia Mateo, Nga Pham, Tam Pham, Matthew Suen, Jennifer Teng (Pharmacy), Vancouver Island Health Authority, Vancouver, BC: Dr Gordon Wood (Lead), Dr Daniel Ovakim (Co-Investigator); Fiona Auld, Gayle Camey, Ralph Fleming, Jennifer Good, Mandeep Manhas (Research Co-ordinators); Karin Boyd, Jane Dheere (Pharmacy), Toronto General Hospital, Toronto, ON: Dr Margaret Herridge (Lead), Dr Eyal Golan, Dr John Granton, (Co-Investigators); Jaimie Archer, Daniel Chen, Brooke Fraser, Cheryl Geen-Smith, Andrea Matte, Priscilia Robles, Cristian Urrea (Research Coordinators); Jane Ascroft, Fatima Haji, Jie Meng, Matthew Suen, Muhammad Walid, Jill Westlund (Pharmacy), Centre intégré universitaire de santé et de services sociaux du Nord-de-l'île-de-Montréal (Hôpital du Sacré-Cœur de Montréal), Montréal, QC: Dr Emmanuel Charbonney (Lead) Dr Yoan Lamarche, Dr Soazig Leguillan, Dr Karim Serri, Dr Colin Verdant, Dr Yanick Beaulieu, Dr Patrick Bellemare, Dr Philippe Bernard, Dr Marc Giasson, Dr Véronique Brunette, Dr Alexandros Cavayas, Dr Émilie Lévesque (Co-Investigators); Halina Labikova, Julia Lainer Palacios, Marie-Ėve Langlois, Virginie Williams (Research Co-ordinators); Thuy Anh Nguyen, Valérie Phaneuf, David Williamson (Pharmacy), Centre intégré universitaire de santé et de services sociaux de l'Estrie-Centre hospitalier universitaire de Sherbrooke, Sherbrooke, QC: Dr François Lamontagne (Lead), Dr Frédérick D'Aragon, Dr Charles St-Arnaud, Dr Hector Quiroz (Co-Investigator); Virginie Bolduc, Elaine Carbonneau, Joannie Marchand, Marie-Hélène Masse (Research Co-ordinators); Sylvie Cloutier, Marianne Guay, Line Morin, Jessie Nicolson, Isabelle Paquette, Patricia Roy, France Théberge (Pharmacy), McGill University Health Centre (Royal Victoria Hospital, Glen Site), Montréal, QC: Dr Arnold S Kristof (Lead), Dr Peter Goldberg, Dr Roupen Hatzakorzian, Dr Sheldon Magder, Dr Jason Shahin, Dr Salman Qureshi (Co-Investigators); Josie Campisi (Research Co-ordinator); Vasilica Botan, Anissa Capilnean, Alyssa Corey, Annick Gagné, Jasmine Mian, Kathleen Normandin (Pharmacy), McGill University Health Centre (Montréal General Hospital), Montréal, QC: Dr Kosar Khwaja (Lead), Dr Dan Deckelbaum, Dr Jeremy Grushka, Dr Ash Gursahaney, Dr David Hornstein, Dr Dev Jayaraman, Dr Tarek Razek, Dr Robert Salasidis, Dr Patrizia Zanelli (CoInvestigators); Norine Alam, Laura Garcia (Research Co-ordinators); Tonia Doerksen, Ariane Lessard, Gilbert Matte, Marie-France Robert (Pharmacy), Centre hospitalier de I'Université de Montréal, Montréal, QC: Dr Martin Girard (Lead); Dr Pierre Aslanian, Dr Sylvain Belisle, Dr François-Martin Carrier, Dr Michaël Chassé, Dr André Denault, Dr Jean-Gilles Guimond, Dr Antoine Halwagi, Dr Paul Hébert, Dr Christopher Kolan, Dr Jordi Mancebo, Dr Nicholas Robillard (Site Co-Investigator); Fatna Benettaib, Dounia Boumahni, Casey Bourdeau Caporuscio, Marie-Ėve Cantin, Virginy Côté-Gravel, Ali Ghamraoui, Martine Lebrasseur, Lancelot Legendre Courville, Stéphanie Lorio, Maria Trinidad Madrid, Nicole Poitras, Romain Rigal, Maya Salame, Valérie Tran (Research Co-ordinators); Katie Bacon, Nathalie Boudreau, Cecilia Carvajal, Lyne Gauthier, Julie Gendron, Karine Jean, Louise Laforest, Antonietta Lembo, Sothun Lim, Jennifer Morrissette, France Pagé, Lucie Pelletier, Marie-Christine Rodrigue (Pharmacy), Royal Alexandra, Edmonton, AB: Dr Jim Kutsiogiannis (Lead), Dr Raiyan Chowdhury, Dr Jon Davidow, Dr Curt Johnston, Dr Richard Johnston, Dr Kim Macala, Dr Sam Marcushamer, Dr Darren Markland, Dr Doug Matheson, Dr Damian Paton-Gay, Dr David Zygun (Co-Investigators); Nadine Grant, Tayne Hewer, Pat Thompson (Research Co-ordinators); Maggie Ge, Janny Hall, Sharon Matenchuk (Pharmacy), Nova Scotia Health Authority QEII, Halifax, NS: Dr Osama Loubani (Lead), Dr Rick Hall, Dr Robert Green (Co-Investigators); Diana Gillis, Lisa Julien, Laura Lee Magennis, Tamara Mitterer (Research Co-ordinators); Joanna Arsenault, Kim Bruce-Payne, Patti Gallant (Pharmacy), Kingston General, Kingston, ON: Dr John Muscedere (Lead), Dr Gord Boyd, Dr Christine D’Arsigny, Dr John Drover, Dr Jason Erb, Dr David Maslove, Dr Chris Parker, Dr Stephanie Sibley (Co-Investigators); Tracy Boyd, llinca Georgescu, Miranda Hunt, Danielle Muscedere (Research Co-ordinators); Cathy Baker, Jennifer Engel, Jennifer Fleming, Lisa Roderick, Shelley Silk, Marcy Spencer, Michelle Tryon (Pharmacy), St Boniface 
Hospital and Health Science Centre, Winnipeg, MB: Dr Ryan Zarychanski (Lead), Dr Marcus Blouw, Dr Kendiss Olafson, Dr Bojan Paunovic, Dr Heather Smith (Co-Investigators); Oliver Gutieror; Justin Lys, Nicole Marten, Sherri Lynn Wingfield (Research Co-ordinators); Marnie Boyle, Halyna Ferens, Debbie Hrabi, Beata Kozak, Chantal MacDonald, Julie Muise (Pharmacy), London Health Sciences CentreVictoria Hospital, London, ON: Dr lan Ball (Lead); Eileen Campbell, Susie Imerovski, Athena Ovsenek, Rebecca Rondinelli (Research Co-ordinators); Teresa Longfield, Amy Moyer, Faith Norris, Janice Sumpton, Karina Teterycz (Pharmacy), Brantford General Hospital, Brantford, ON: Dr Brenda Reeve (Lead); Karen Bento, Megan Davis, Will Dechert, Krista Gallo, Barbara Longo, Courtney Mullen, Elysia Skrzypek, Laurenne Wierenga (Research Co-ordinators); Wesam Abuzaiter, Lynda Amorim, Rosemarie Bauer, Rachel Damota, Thoa Ho, Nicole Macdougall, Mary Thornewell, Lara Pe, Jennifer Visocchi (Pharmacy), Health Sciences Centre, Winnipeg, MB: Dr Ryan Zarychanski (Lead), Dr Bojan Paunovic (Co-Investigator); Justin Lys, Nicole Marten (Research Co-ordinators); Audrey Bhairo, Halyna Ferenes, Beata Kozak, Debra Kubin, Dawn-Lee McLaughlin, Maria Valente (Pharmacy), St Joseph's Hospital, Toronto, ON: Dr Rob Cirone (Lead), Dr Jennie Johnstone (Co-Investigator); Kanthi Kavikondala, Axelle Pellerin, Laura Tomat (Research Co-ordinators); Jeff Carter, Jiten Jani, Brendan Yeats (Pharmacy), Royal Columbia Hospital, New Westminster, BC: Dr Steve Reynolds (Lead); Suzette Willems (Research Coordinator); Tina Sekhon (Pharmacy), William Osler Hospital, McKenzie Health, Brampton Civic Hospital Brampton, ON: Dr Sebastien Trop (Lead), Dr Alexandra Binnie, Dr Ronald Heslegrave (Co-Investigator); Kim Sharman, Zaynab Panchbhaya (Research Co-ordinators); Rakhi Goel, Kim Kozluk, Julianne Labelle, Hina Marsonia, Cecillia Scott (Pharmacy), London Health Sciences Centre-University Hospital, London, ON: Dr Dave Nagpal (Lead), Dr lan Ball (Co-Investigator); Tracey Bentall, Jessica Sturt-Smith (Research Co-ordinators); Michelle Alexander, Tammy Ellis, Mindy Muylaert, Cindy Paczkowski (Pharmacy), University of Alberta, Edmonton, AB: Dr Wendy Sligl (Lead), Dr Sean Bagshaw (Co-Investigator); Nadia Baig, Lorena McCoshen (Research Co-ordinators); Katrina Alexandropoulos, Sherri Bain, Michelle Brandt, Cathy Constable, Kari Douglas, Shaleen Maharaj, Sabrina Travers (Pharmacy), Foothills Hospital, University of Calgary, Calgary, AB: Dr Tom Stelfox (Lead), Dr Philippe Couillard, Dr. Christopher Doig, Dr Ken Parhar (Co-Investigators); Joshua Booth, Cassidy Codan, Stacy Ruddell (Research Co-ordinators); Candice Cameron, Rhonda Edison, Anne Martin, Breanna Mina (Pharmacy), Peter Lougheed, University of Calgary, Calgary, AB: Dr Dan Niven (Lead), Dr Luc Berthiaume, Dr Jonathan Gaudet (Co-Investigators); Joshua Booth, Cassidy Codan, Gina Fleming, Katie Ross, Stacy Ruddell (Research Co-ordinators); Candice Cameron, Rhonda Edison, Anne Martin, Breanna Mina (Pharmacy), Niagara Health, St Catharines, ON: Dr Jennifer Ly Tsang (Lead), Dr Erick Duan (Co-Investigator); Mercedes Carmargo, Beverly Hoekstra (Research Co-ordinators); Rita Caporuscio, Rachel Kressner Falvo, Dimitra Fleming, Carmelina Maxwell, Karmen Plantic (Pharmacy), Centre intégré universitaire de santé et de services sociaux de l'Est-de-l'Île-de-Montréal (Hôpital Maisonneuve-Rosemont), Montréal, QC: Dr François Marquis (Lead), Dr Han Tin Wang, Dr Francis Toupin, Dr Stephane Ahern, Dr Brian Laufer, Dr Marc Brosseau (Co-Investigators); Pauline Dul, Johanne Harvey, Lotthida Inthanavong, Danae Tassy (Research Co-ordinators); Helen Assayag, Maude Bachand, Marysa Betournay, Karine Daoust, Kristine Goyette, Ariane Lessard, Marceline Quach (Pharmacy), Grand River Hospital, Kitchener, ON: Dr Paul Hosek (Lead), Dr Bill Plaxton (Co-Investigator); Catherine Armstrong, Rhonda Barber, William Dechert, Janelle Ellis, Kayla Fisk, Melissa Gabnouri, Emilie Gordon, Rebecca Haegens, Lisa Halford, Brooklynn Hillis, Rebecca Jesso, Jenn McLaren, Elliot McMillan, Mariska Pelkmans, Matthew Rekman, Sylvia Sinkovitis, Monica Truong, Michelle White (Research Co-ordinators); Noah Bates, Susan Bryden-Cromwell, Lisa Cha, Colleen Cameron, Aminah Deen, Sheri DiGiovanni, Anders Foss, Esther Lee, Heidi MacGregor, Esther Galbraith Robyn McArthur, Julie McGregor, Keith Miller, Sharon Morris, Shelley Parker, Candice Smith, Joanna Stoglow, Jennifer Tung, Melissa Vos (Pharmacy), Sunnybrook Health Sciences Centre, Toronto, ON: Dr Neill Adhikari (Lead), Dr Andre Amaral, Dr Andre Carlos, Dr Brian Cuthbertson, Dr Rob Fowler, Dr Damon Scales (Co-Investigators); Navjot Kaur, Nicole Marinoff, Adic Perez, Jane Wang (Research Co-ordinators); Katrina Hatzifilalithis, John lazzetta, Chrys Kolos, Ingrid Quinton (Pharmacy), Joseph Brant Hospital, Burlington, ON: Dr Paul Lysecki (Lead), Dr Joseph Berlingieri, Dr Sameer Shaikh, Dr Steven Skitch (Co-Investigators); Tracy Campbell (Research Co-ordinator); Hala Basheer, Kathy Bruder, Jane Cheng, Kaiser Qureshi, Celeste Thibault (Pharmacy), Institut Universitaire de Cardiologie et de Pneumologie de Québec, Québec, QC: Dr François Lellouche (Lead), Dr Ying Tung Sia, Dr Mathieu Simon (Co-Investigators); Pierre-Alexandre Bouchard, Patricia Lizotte (Research Co-ordinators); Nathalie Chateauvert, Thérèse Grenier (Pharmacy), Centre intégré de santé et de services sociaux de Chaudière-Appalaches (L'Hôtel-Dieu de Lévis), Lévis, QC: Dr Patrick Archambault (Lead), Dr Jean-François Bellemare, Dr Simon Bordeleau, Dr Christine Drouin, Dr Benoît Duhaime, Dr Ann Laberge, Dr Philippe Lachance (Co-Investigators); Mélanie Constantin, Estel Deblois,
Maude Dionne, Lise Lavoie, Isabelle Michel, Alexandre Pépin, Sandrine Poulin (Research Co-ordinators); Sarah Anctil, Amélie Chouinard, Louis-Étienne Marchand, Robin Roy (Pharmacy). USA—Mayo Clinic, Rochester, MN: Dr Rodrigo Cartin-Ceba (Lead), Dr Richard Oeckler (Co-Investigator); Brenda Anderson, Lavonne Liedl, Laurie Meade, Sueanne Weist (Research Co-ordinators); Anna Bartoo, Debbie Bauer, Vince Brickley, Shaun Bridges, Greg Brunn, Jennifer Eickstaedt, Jill Randolph, Sandy Showalter, Erin Stern, Melissa Wendling (Pharmacy), St John's Mercy Medical Center, St Louis, M0: Dr Robert Taylor (Lead); Margaret Cytron, Kim Fowler, Katie Krause, Jackie 0'Brien, Marianne Tow (Research Co-ordinators); John Ma, Kaitlin Stassi (Pharmacy). Saudi Arabia—King Abdulaziz Medical Center, Riyadh, Saudi Arabia: Dr Yaseen Arabi (Lead), Dr Abdulaziz Al-Dawood, Dr Haytham Tlayjeh, Dr Alaaeldien Ghanem, Dr Ahmad Hassanien, Dr Mohamed Hegazy, Dr Ashraf El Sharkawi (Co-Investigators); Felwa Bin Humaid, Hala Alanizi, Nadyah Alanizy, Njoud Al Bogami (Research Co-ordinators); Dr Mohammed Muhaidib, Dr Jawaher Gramish, Randa Alsomali, Nora Devera, Marjane Villafranca (Pharmacy).

Contributors Conception and design: VIL, DJC, BR, FX, JJ, RF. Manuscript writing: VIL, DJC, BR, FX, JJ, RF. Critical revision and final approval of the manuscript: VIL, DJC, BR, FX, JJ, RF, JB, FL, JCM, LT, DH-A. Obtained funding: DJC, JJ, FL, JCM, LT. Administrative, technical or material support: FX, BR, DJC, JJ, DH-A, LT. Study supervision: VIL, DJC, BR, FX, JJ, RF. All authors read and approved the final manuscript.

Funding This economic evaluation (E-PROSPECT) and PROSPECT is funded by the CIHR, McMaster University, St. Joseph's Healthcare Hamilton, Canadian Frailty Network, Physicians Services Incorporated of Ontario, the Hamilton Academic Health Sciences Organization and the Academic Medical Organization of Southwestern Ontario. DJC is a Canada Research Chair of the Canadian Institutes of Health Research. Study products were donated by i-Health, Inc., the manufacturers of $L$. rhamnosus $\mathrm{GG}$.

\section{Competing interests None declared.}

Patient consent for publication Not required.

Provenance and peer review Not commissioned; externally peer reviewed.

Open access This is an open access article distributed in accordance with the Creative Commons Attribution Non Commercial (CC BY-NC 4.0) license, which permits others to distribute, remix, adapt, build upon this work non-commercially, and license their derivative works on different terms, provided the original work is properly cited, appropriate credit is given, any changes made indicated, and the use is non-commercial. See: http://creativecommons.org/licenses/by-nc/4.0/.

\section{ORCID iDs}

Vincent Issac Lau http://orcid.org/0000-0002-9939-7348

Bram Rochwerg http://orcid.org/0000-0002-8293-7061

\section{REFERENCES}

1 Muscedere JG, Martin CM, Heyland DK. The impact of ventilatorassociated pneumonia on the Canadian health care system. $J$ Crit Care 2008;23:5-10.

2 Safdar N, Dezfulian C, Collard HR, et al. Clinical and economic consequences of ventilator-associated pneumonia: a systematic review. Crit Care Med 2005;33:2184-93.

3 Muscedere J, Sinuff T, Heyland DK, et al. The clinical impact and preventability of ventilator-associated conditions in critically ill patients who are mechanically ventilated. Chest 2013;144:1453-60.

4 Muscedere J, Dodek P, Keenan S, et al. Comprehensive evidencebased clinical practice guidelines for ventilator-associated pneumonia: prevention. J Crit Care 2008;23:126-37.

5 Food and Agriculture Organization of the United Nations, World Health Organization. Joint FAO/WHO working group report on drafting guidelines for the evaluation of probiotics in food, 2002. Available: https://www.who.int/foodsafety/fs_management/en/ probiotic_guidelines.pdf [Accessed 23 Nov 2019].

6 WHO. Diarrhoea. Available: http://www.who.int/topics/diarrhoea/en/ [Accessed 17 Mar 2019].

7 Marshall JC. Gastrointestinal flora and its alterations in critical illness. Curr Opin Clin Nutr Metab Care 1999;2:405-11.

8 Stappenbeck TS, Hooper LV, Gordon Jl. Developmental regulation of intestinal angiogenesis by indigenous microbes via Paneth cells. Proc Natl Acad Sci U S A 2002;99:15451-5.

9 Tanoue T, Honda K. Induction of Treg cells in the mouse colonic mucosa: a central mechanism to maintain host-microbiota homeostasis. Semin Immunol 2012;24:50-7. 
10 Brenchley JM, Douek DC. Microbial translocation across the GI tract. Annu Rev Immunol 2012;30:149-73.

11 Hao Q, Lu Z, Dong BR, et al. Probiotics for preventing acute upper respiratory tract infections. Cochrane Database Syst Rev 2011:CD006895.

12 Hempel S, Newberry SJ, Maher AR, et al. Probiotics for the prevention and treatment of antibiotic-associated diarrhea: a systematic review and meta-analysis. JAMA 2012;307:1959-69.

13 Ritchie ML, Romanuk TN. A meta-analysis of probiotic efficacy for gastrointestinal diseases. PLoS One 2012;7:e34938.

14 Johnson S, Maziade P-J, McFarland LV, et al. Is primary prevention of Clostridium difficile infection possible with specific probiotics? Int $J$ Infect Dis 2012;16:e786-92.

15 Manzanares W, Lemieux M, Langlois PL, et al. Probiotic and synbiotic therapy in critical illness: a systematic review and metaanalysis. Crit Care 2016;19:262.

16 Goldenberg JZ, Yap C, Lytvyn L, et al. Probiotics for the prevention of Clostridium difficile-associated diarrhea in adults and children. Cochrane Database Syst Rev 2017;12:CD006095.

17 Lau VI, Rochwerg B, Xie F, et al. Probiotics in hospitalized adult patients: a systematic review of economic evaluations. Can J Anaesth 2020;67:247-61.

18 Cook DJ, Johnstone J, Marshall JC, et al. Probiotics: Prevention of Severe Pneumonia and Endotracheal Colonization Trial-PROSPECT: a pilot trial. Trials 2016;17:377.

19 Johnstone J, Meade M, Marshall J, et al. Probiotics: Prevention of Severe Pneumonia and Endotracheal Colonization TrialPROSPECT: protocol for a feasibility randomized pilot trial. Pilot Feasibility Stud 2015;1:19.

20 Johnstone J, Heels-Ansdell D, Thabane L, et al. Evaluating probiotics for the prevention of ventilator-associated pneumonia: a randomised placebo-controlled multicentre trial protocol and statistical analysis plan for PROSPECT. BMJ Open 2019;9:e025228.

21 Drummond MF, Sculpher MJ, Claxton K, et al. Methods for the economic evaluation of health care programmes. Oxford University Press, 2015.

22 Sanders GD, Neumann PJ, Basu A, et al. Recommendations for conduct, methodological practices, and reporting of costeffectiveness analyses: second panel on cost-effectiveness in health and medicine. JAMA 2016;316:1093-103.

23 Husereau D, Drummond M, Petrou S, et al. Consolidated Health Economic Evaluation Reporting Standards (CHEERS) statement. BMJ 2013;346:f1049.

24 Polk RE, Fox C, Mahoney A, et al. Measurement of adult antibacterial drug use in 130 US hospitals: comparison of defined daily dose and days of therapy. Clin Infect Dis 2007;44:664-70.

25 Kuster SP, Ruef C, Ledergerber B, et al. Quantitative antibiotic use in hospitals: comparison of measurements, literature review, and recommendations for a standard of reporting. Infection 2008;36:549-59.

26 Little RJA, Rubin DB. Statistical analysis with missing data. New York, NY, USA: John Wiley \& Sons, Inc, 1986

27 Zhang Y, Alyass A, Vanniyasingam T, et al. A systematic survey of the methods literature on the reporting quality and optimal methods of handling participants with missing outcome data for continuous outcomes in randomized controlled trials. J Clin Epidemiol 2017;88:67-80.
28 Fowler RA, Mittmann N, Geerts WH, et al. Economic evaluation of the prophylaxis for thromboembolism in critical care trial (E-PROTECT): study protocol for a randomized controlled trial. Trials 2014;15:502.

29 Bank of Canada. Bank of Canada inflation calculator. Available: https://www.bankofcanada.ca/rates/related/inflation-calculator/ [Accessed 12 Apr 2019].

30 World Bank. Official exchange rate (LCU per US\$, period average). Available: https://data.worldbank.org/indicator/PA.NUS.FCRF [Accessed 11 Feb 2019].

31 OANDA. Currency converter | foreign exchange rates. Available: https://www.oanda.com/currency/converter/ [Accessed 23 Mar 2019].

32 Tukiainen M. RatesFX-daily foreign exchange rates, information about currencies and currency markets. Available: https://www. ratesfx.com/ [Accessed 11 Feb 2019].

$33 \mathrm{Ng} \mathrm{R}$, Hasan B, Mittmann N, et al. Economic analysis of NCIC CTG JBR.10: a randomized trial of adjuvant vinorelbine plus cisplatin compared with observation in early stage non-small-cell lung cancer-a report of the working group on economic analysis, and the lung disease site group, National Cancer Institute of Canada Clinical Trials Group. J Clin Oncol 2007;25:2256-61.

34 Fowler RA, Mittmann N, Geerts W, et al. Cost-effectiveness of dalteparin vs unfractionated heparin for the prevention of venous thromboembolism in critically ill patients. JAMA 2014;312:2135-45.

35 Patel PJ, Singh SK, Panaich S, et al. The aging gut and the role of prebiotics, probiotics, and synbiotics: a review. J Clin Gerontol Geriatr 2014;5:3-6.

36 Wachholz PA, Boas PJFV, dos Santos Nunes V, et al. Evidence on the role of prebiotics, probiotics, and synbiotics in gut health and disease prevention in the elderly. J Gerontol Geriatr 2014;5:1-2.

37 Bagshaw SM, Stelfox HT, McDermid RC, et al. Association between frailty and short- and long-term outcomes among critically ill patients: a multicentre prospective cohort study. CMAJ 2014:186:E95-102.

38 Fieller EC. Some problems in interval estimation. J R Stat Soc Series B 1954;16:175-85.

39 Doubilet P, Begg CB, Weinstein MC, et al. Probabilistic sensitivity analysis using Monte Carlo simulation. A practical approach. Med Decis Making 1985;5:157-77.

40 Wilcox ME, Vaughan K, Chong C, et al. Cost-effectiveness studies in the ICU: a systematic review. Crit Care Med 2019.

41 Lenoir-Wijnkoop I, Nuijten MJC, Craig J, et al. Nutrition economic evaluation of a probiotic in the prevention of antibiotic-associated diarrhea. Front Pharmacol 2014:5:13.

42 Vermeersch SJ, Vandenplas $\mathrm{Y}$, Tanghe A, et al. Economic evaluation of S. boulardii CNCM I-745 for prevention of antibiotic associated diarrhoea in hospitalized patients. Acta Gastroenterol Belg 2018;81:269-76.

43 Kerlin MP, Cooke CR. Understanding costs when seeking value in critical care. Ann Am Thorac Soc 2015;12:1743-4.

44 Krahn M, Bryan S, Lee K, et al. Embracing the science of value in health. CMAJ 2019;191:E733-6.

45 Vijayaraghavan BKT, Willaert X, Cuthbertson BH. Cost-effectiveness analysis should be mandatory in clinical-effectiveness research. CMAJ 2019;191:E1140. 